\title{
Data interaction model for power transaction based on CIM
}

\author{
Dexiang Jia ${ }^{1}$, Yongwei Liu $^{2}$, Yu Zang ${ }^{1}$, Qingqi Chen ${ }^{2}$, Yanhong Zhou ${ }^{2}$, and Ganyun $\mathrm{Lv}^{1, *}$ \\ ${ }^{1}$ State Grid Energy Research Institute Co., LTD, 102209, Beijing China \\ ${ }^{2}$ State Grid Hunan Electric Power Co., Ltd., 410004, Changsha China
}

\begin{abstract}
With the increase of power transaction business, traditional power transaction data interaction models cannot fully meet the needs of data interaction. The data model conforming to the power transaction data architecture plays an important role. Data interaction requirements of Chinese electricity market transaction business are analyzed, and the specific method of power transaction data description is given in the IEC61970 protocol standard. Also a common information model (CIM) for electricity trans-action data interaction is built that conforms to the data interaction architecture. Finally the functional description of the model is built adopting the unified modeling language (UML). Compared with the existing electricity transaction data interaction model, the method is beneficial to reduce the degree of data redundancy, increase the speed of data interaction, and thus improve the transaction efficiency of the electricity market.
\end{abstract}

\section{Introduction}

As the deep reform of the power system, the process of power marketization in China is accelerating, and the continuous increase in power transaction business has led to more frequent interactions of power transaction data ${ }^{[1]}$. Thus the power trading platforms need to have more rapid responsiveness ${ }^{[2]}$. The current power trading platform business mainly involves core businesses such as power commodity trading, market settlement, and auxiliary businesses such as market services and market analysis ${ }^{[3]}$. The data between many businesses is closely linked, and data interaction models are required to meet the data interaction needs of power transaction services.

The current research on the data interaction model of the power trading platform focuses on how to support the massive transaction data interaction by strengthening the construction of the platform architecture, and there is little research on the transaction data model itself. Cheng $\operatorname{Lin}^{[4]}$ studied the supporting role of micro-service architecture ${ }^{[5]}$ for power transaction platforms, but lacked thinking about specific power transaction business data. Literature[6] describes the architecture of the power trading platform based on the microservices and adopts the idea of hierarchical system design. Chen Xiaoyong ${ }^{[7]}$ used the middle-office architecture not only to have the characteristics of a micro-service

\footnotetext{
*Corresponding author: 16702324@qqq.com
} 
architecture, but also to quickly process operating data to achieve strong support for the power transaction business. Literature[8-9] adopted the idea of hierarchical, categorized and interactive power transaction data to support the power transaction business. Literature[10] uses the Public Cloud + Private Cloud solution of the US PJM power market to implement data storage operations. Zheng Yaxian ${ }^{[1]}$ combined with the IEC-62325 standard model to build a multi-level market data model for power transaction data.

Most of studies solve the current problems of power transaction data interaction from the perspective of system platform architecture, but there are fewer ideas for research from the perspective of data itself. The IEC-61970 protocol standard ${ }^{[12]}$ proposes a series of power data Framework models, including the common information model ${ }^{[13]}(\mathrm{CIM})$ for power data interaction, and there are many ways to describe the data model, the most important ones include Extensible Markup Language ${ }^{[14]}(\mathrm{XML})$, resource description framework $^{[15]}(\mathrm{RDF})$ and Unified Modeling Language ${ }^{[16]}(\mathrm{UML})$, etc. The description methods of these data models are mainly different in the way of model description.

This article start from the model of the data itself, analyze the interactive data requirements involved in the current transaction process, and combine the IEC61970 standard protocol framework to construct a transaction data common information model (CIM) that conforms to the power transaction data transmission architecture, and adopts a unified modeling language (UML). The model is to reduce the degree of redundancy in the transaction data transmission, and improve speed and quality of data interaction process under the premise of the continuous growth of the current transaction business.

\section{Common information model protocol framework on IEC-61970}

The IEC-61970 standard is the International Electrotechnical Commission's logical model definition for grid operation. It describes a series of frameworks such as data processing and model establishment involved in processes such as grid operation control and energy trading. The data information involved in the power transaction process not only includes the data involved in the IEC-61970 standard, but also includes IEC-61968 and IEC-62325.

The common information model (CIM) has strong interoperability and independence. On the one hand, through its unique association method, it provides a general model for the information expression of electric power companies. On the other hand, CIM can be independent of a single protocol standard and build a single protocol standard model. CIM divides data through "class attributes" and realizes the establishment of data models through inheritance, association, and aggregation. The clearest way to express CIM is to describe the model through the Unified Modeling Language (UML) as shown in Figure 1.

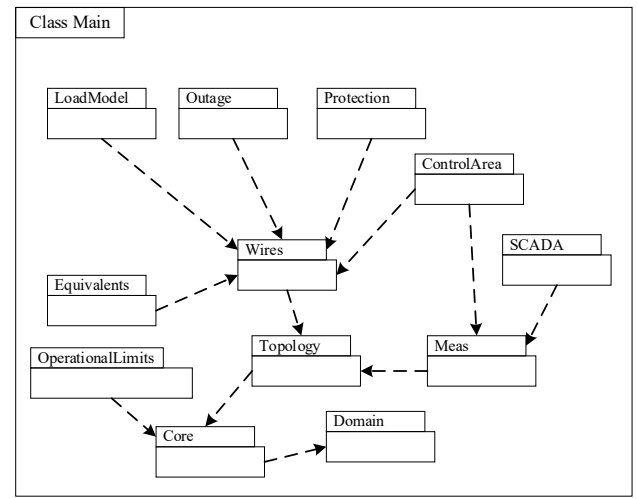

Fig. 1. UML description of common information model. 
The method of hierarchically dividing data according to "class attributes" shown in Figure 1 is beneficial to the overall call of data in the power transaction process and can reduce the redundancy problem of the actual data transmission process.

\section{Data interaction analysis of power transaction business}

The actual electricity trading process requires coordination among the various systems of the electricity trading platform. The electricity trading platform serves as the carrying platform for the electricity trading business. The complete electricity trading business process is shown in Figure 2.

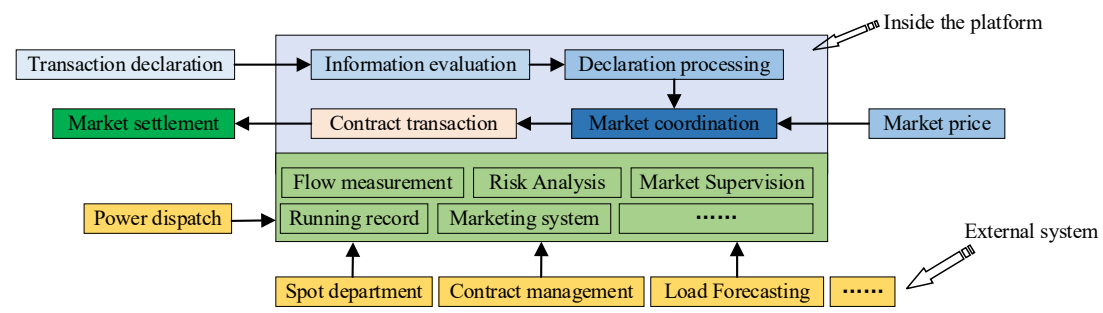

Fig. 2. Simplified process for power transaction.

As shown as Figure 2, transaction members first need to declare their business needs, and the transaction platform internally performs the declaration processing from member information evaluation and transaction information review, etc., and then the transaction center combines the spot market quotation for market coordination, and then the transaction is concluded. Transactions are carried out on the basis of contracts, and finally market settlement is carried out through the settlement system. In addition to declaration, quotation and settlement, the power trading center's flow measurement, risk analysis, market supervision and scheduling, spot contract management, load forecasting and other systems jointly support the entire process of power trading business.

The classification of the systems participating in the entire power transaction business process is conducive to further research on the specific interactive information between the systems in the power transaction process, and has a positive impact on reducing the redundant data in the transaction process. Combining with the system support involved in the power transaction business transmission process shown in Figure 2, each system is divided into the power trading platform's own system and the external system, and the relationship between the systems is shown in Figure 3.

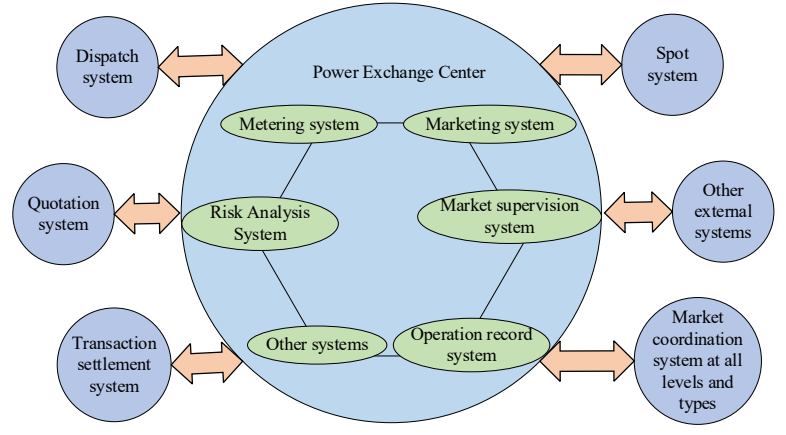

Fig. 3. Diagram of the power transaction platform system.

The internal system of the power trading center mainly includes the electric energy measurement system, the marketing system, the risk analysis system, the market 
supervision system, and the operation record system, while the external system includes the market quotation system, the spot system, the power dispatch system, the market coordination system at all levels, Transaction settlement system and other systems that support power trading business. In the process of power trading business, massive data transmission is required between the trading center and external systems and between internal systems to complete power trading.

Taking the internal data transmission of the power trading center as an example, the main information transmitted between the systems is discussed. The power transaction business data is extremely large. In the actual transaction process, a powerful platform architecture is required to support the operation of the data. Taking into account that some information needs to be interacted multiple times between different systems, if this type of data is sent to the system that carries the data through the platform during each actual interaction, the system that carries the data will respond and send it to the demand side, it will cause a large amount of data redundancy and increase the data carrying capacity of the platform architecture, thereby reducing the speed of power transactions and reducing the timeliness of data.

On the basis of considering the above problems, this paper adopts the idea of CIM for data classification modeling, constructs a data model suitable for data transmission in the power transaction process, and describes it through UML.

\section{Power transaction data interaction model based on CIM}

In the process of establishing the common information model of electric power data, firstly, the electric power data should be classified according to different categories. On the basis of the model classification, the specific information contained in the classification should be studied, and a unified model name and data type should be established for the specific information, physical library tables and physical fields and other unified symbols to build a common information model that is easy to transfer.

According to the electricity transaction process, this paper divides the involved data into basic, dispatch, transaction and settlement models. Part of the information contained in various models is shown in Figure 4.

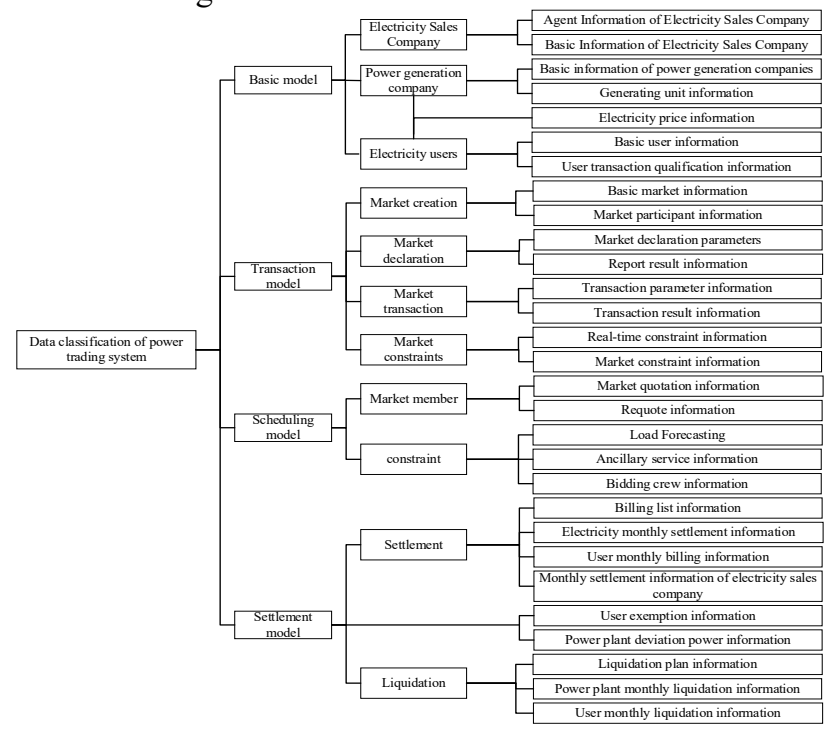

Fig. 4. Classification of power transaction business data. 
Taking basic data as an example, basic data includes three aspects of information about electricity sales companies, power generation companies, and users. It mainly includes basic information of all parties, agent information of electricity sales companies, generator set information, and electricity price information.

The categories such as power plant information, user information, and trading platform information also include basic information that belongs to this category. The electricity price information in the basic data is established as an example, combined with the IEC61970 protocol standard for energy transaction management, shown as Table 1.

Table 1. Electricity price information database table.

\begin{tabular}{cccc}
\hline Unified model name & type & Description & Physical library table \\
\hline companyid & string & System number & b_priceinfo \\
companyname & string & system name & b_priceinfo \\
accountid & string & Marketing account number & b_priceinfo \\
mataringid & string & Measuring point number & b_priceinfo \\
pricetype & number & Electricity price category & b_priceinfo \\
wetdrytype & number & Rich and dry type & b_priceinfo \\
timetype & number & Time period type & b_priceinfo \\
begdate & date & effective date & b_priceinfo \\
enddate & date & deadline & b_priceinfo \\
\hline
\end{tabular}

According to the CIM method of constructing a data model, combined with the data information shown in Table 1 and Table 2, a common information model of electricity price information is established, and the UML description as shown in Figure 5.

Each label in Figure 5 contains a unified model name, data type, model name description and its corresponding physical library table, etc., which facilitates the accurate extraction of effective information during the transaction process and reduces the time delay in the transaction process.

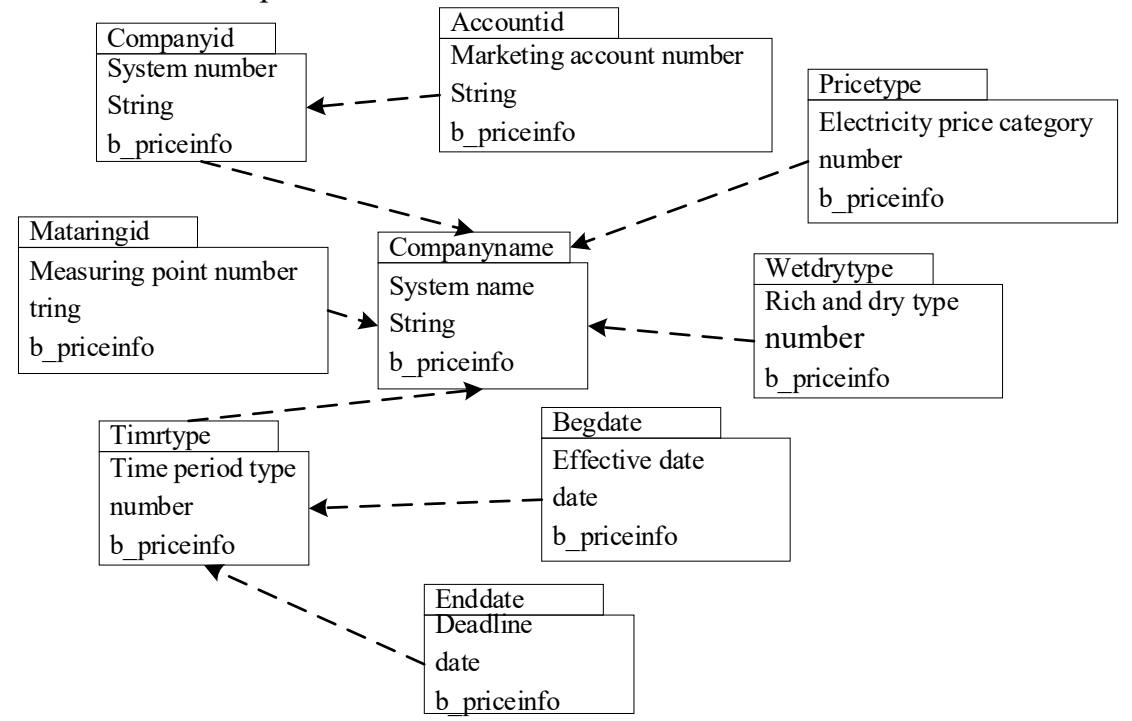

Fig. 5. Common Information Model of Electricity Price Information.

According to the above ideas, combined with the classification of data information involved in the power transaction business process, each data common information model can be established for different data information classifications. The power transaction center can build a database table corresponding to the model based on the established common information model. Relying on the data interaction architecture of the power 
trading platform will help speed up the extraction of data and information in the power trading business process and improve the efficiency of the power trading business.

\section{Conclusion}

The current research on the optimization of power trading business focuses on the optimization and upgrading of the trading system platform architecture. Starting from the data ontology involved in the power trading process, this article studies the process of data interaction in the power trading process and the types of data participating in the power trading process. It is classified, combined with IEC61970 and two other power data-related standards, analyzes the construction principles of common information models, conducts common information modeling for power transaction business data, and uses UML to describe the model.

The common information model of electricity transaction data built in this paper is beneficial to reduce the redundancy of data information interaction in the transaction process, shorten the time of the electricity transaction process, and improve the efficiency of the electricity transaction platform for the electricity transaction business.

This work was supported by Science and Technology Foundation of SGCC "Key Technologies for Operation Optimization of New Generation Power Trading Platform"

\section{References}

1. L.Ma, M.H.Fan, H.Y. Qu, et al. Electric Power ,53, 9(2020)

2. S.N.Zhang, Y.W.Liu, W.L.Hu, et al. Power System Technology.(to be published)

3. L.J.Shi, P.Shao, X.Zhang, et al. Automation of Electric Power Systems, 41, 10(2017)

4. L.Cheng, H.N.Wang, C.C.Gao. Power System Technology,42, 6(2018)

5. Z.Y.Feng, Y.W.Xu, X.Xue, et al. J Comp. Res. Dev,57,20(2020)

6. W.X.Zhan, Z.X.Zhang, L.Du, et al. Ecologically Interconnected Digital Power-2019 Electric Power Industry Informatization Annual Conference. 4 (2019)

7. X.Y.Chen, H.Y.He, L.Huo. Digital Technology \& Application.36,11(2018)

8. J.C Shang. Automation of Electric Power Systems,34, 7(2010)

9. J.C Shang. Automation of Electric Power Systems,34, 5(2010)

10. J.S.Zhu, M.Z.Wang, L.P.Yang, et al. Railway Computer Application,11, 4(2015)

11. Y.X.Zheng, Z.L.Yang, B.K.Xue, et al. Automation of Electric Power Systems, 39, $8(2015)$

12. S.X.Wang, Z.H.Meng, S.C.Yuan. Inter. J. Elec. Power. Energy Sys, 118(2020)

13. Y.Ding, M.Ding, R.Bi, et al. Power System Protection and Control, 38, 6(2010)

14. C.X.Niu, W.Song, K.F.Zhang. Electric Power Automation Equipment, 07, 4(2006)

15. C.Yang, J.M.Lu, J.Feng. J. Comp. Appl, 40, 8(2020)

16. D.Liu, Y.P.Chen, G.Shen, et al. High Voltage Engineering,06, 5(2006) 